\title{
Comparison of Half and Full Doses of an MF59- Adjuvanted Cell Culture-Derived A/H1N1v Vaccine in Japanese Children
}

Yuji Yasuda · Ryoya Komatsu · Kenji Matsushita · Taketsugu Minami · Yutaka Suehiro · Hiroshi Sawata . Noriko Nakura $\cdot$ Ralf K. Jaeger · Maria Lattanzi

Received: May 7, 2010 / Published online: June 25, 2010

(c) The Author(s) 2010. This article is published with open access at Springerlink.com

\section{ABSTRACT}

Introduction: The substantial pandemic (A/H1N1v) influenza disease burden in children highlights the need for effective vaccination. We report the results of modern cell culture technology, lower doses of antigen, and different doses of MF59 ${ }^{\circledR}$ adjuvant (Novartis Vaccines, Marburg, Germany), on the immunogenicity

Yuji Yasuda

Yasuda Clinic, Minami-ku, Kyoto, Japan

Ryoya Komatsu

Ryoya Komatsu Clinic, Rinku Kita, Izumisano,

Osaka, Japan

Kenji Matsushita

Kamoike Seikyou Clinic, Kamoike, Kagoshima, Japan

Taketsugu Minami

Minami Clinic, Kamoike, Kagoshima, Japan

Yutaka Suehiro

Saiseikai Nakatsu Hospital, Kita-ku, Osaka, Japan

Hiroshi Sawata $\cdot$ Noriko Nakura

Novartis Pharma, Minato-ku, Tokyo, Japan

Ralf K. Jaeger

Novartis Vaccines and Diagnostics, Marburg, Germany

Maria Lattanzi $(\bowtie)$

Clinical Development, Novartis Vaccines and

Diagnostics, Via Fiorentina 1, 53100 Siena, Italy.

Email: maria.lattanzi@novartis.com and safety profile in a healthy Japanese pediatric population. Methods: A total of 123 children from 6 months to 19 years of age were randomly assigned in a 1:1 ratio to receive, at 21-day intervals, two doses of either $3.75 \mu \mathrm{g}$ antigen with $50 \%$ of the standard MF59 dose (group A) or $7.5 \mu \mathrm{g}$ antigen and 100\% standard MF59 dose (group B). Antibody levels were measured by hemagglutinin inhibition (HI) and microneutralization assays on day 1 and on days 22 and 43 (3 weeks after the first and second vaccinations, respectively). Solicited adverse events were reported for 7 days after each injection and spontaneous events were reported throughout the study period. Results: At 3 weeks after the first vaccination, seroprotective HI antibodies (titers $\geq 40$ ) were observed in $56 \%$ and $78 \%$ of subjects from groups A and B, respectively; $100 \%$ in both groups exhibited HI titers $\geq 40$ after the second dose. The reactogenicity profile was acceptable, with local and systemic reactions described as mainly mild to moderate in severity. Five serious adverse events were reported, but none related to the study vaccine. Conclusion: One dose of cell culture-derived $\mathrm{A} / \mathrm{H} 1 \mathrm{~N} 1 \mathrm{v}$ vaccine containing $7.5 \mu$ g antigen with the full MF59 adjuvant dose was immunogenic and well tolerated in healthy 
Japanese children, meeting all three European Union Committee for Medicinal Products for Human Use (EU CHMP) licensure criteria. Two doses of $3.75 \mu \mathrm{g}$ antigen with $50 \%$ of the standard MF59 dose fulfilled these licensure criteria.

Keywords: cell culture vaccine; children; A/H1N1v influenza; MF59 adjuvant

\section{INTRODUCTION}

In the spring of 2009, a novel influenza A $(\mathrm{A} / \mathrm{H} 1 \mathrm{~N} 1 \mathrm{v})$ virus appeared in Mexico and the US, and its resultant spread around the globe has been classified as the first pandemic of the twentyfirst century. ${ }^{1,2}$ As of January 31, 2010, A/H1N1v has been identified in humans in more than 209 countries and caused at least 15,174 deaths. ${ }^{3}$ In Japan, a total of 16,570 hospitalizations and 180 fatal cases have been reported. ${ }^{4}$ In contrast to peak periods of seasonal influenza, when influenza hospitalizations are more common among persons 65 years of age or older and those under the age of 5 years, more than $85 \%$ of the hospitalizations in Japan occurred in people younger than 20 years. ${ }^{4}$ This trend of high percentages of hospitalization in children may be explained by a lack of pre-existing immunity against the new A/H1N1v virus and enhanced transmission opportunities in the pediatric population. ${ }^{5}$ In previous studies, influenza vaccination in children has been shown to reduce their infection rate as well as the virus's transmission among families and the community. ${ }^{6,7}$ In addition, mass vaccination of children may contribute to controlling A/H1N1v pandemic influenza. ${ }^{8}$

Although previous experience with other pandemic vaccines has suggested that two doses of vaccines for children and adults would be needed to induce protection against the new A/H1N1v virus strain, ${ }^{9}$ the results from recently published trials in adults have consistently demonstrated satisfactory immune responses after the administration of only one dose of vaccine. ${ }^{10-15}$ In children, available data are less consistent, some studies indicating that a twodose administration schedule is needed to provide protection in younger children, ${ }^{12,13,15}$ while others report effective responses after one dose in children aged 6 months to 9 years ${ }^{16}$ and 3 to 17 years. ${ }^{17}$ In the latter study, favorable outcomes were reported for a $7.5 \mu \mathrm{g}$ dose of A/H1N1v vaccine with MF59 ${ }^{\circledR}$ adjuvant (Novartis Vaccines, Marburg, Germany) compared with $15 \mu \mathrm{g}$ and $30 \mu \mathrm{g}$ doses without adjuvant in children 3 to 8 years of age. ${ }^{17}$ Appropriate adjuvants may significantly enhance and broaden the immune response even with reduced antigen content, ${ }^{18-20}$ with the additional benefit of improved cross-reactivity against viral mutations, ${ }^{18,20,21}$ a characteristic of particular importance in later phases of a pandemic. The use of such adjuvants in 2009 A/H1N1v vaccines has been suggested by the World Health Organization (WHO). ${ }^{22}$

There are well documented health disparities and increased morbidity and mortality rates from influenza among specific ethnic populations during the current and previous pandemics. ${ }^{23}$ It is therefore important to assess the immunogenicity and safety profiles of $\mathrm{A} / \mathrm{H} 1 \mathrm{~N} 1 \mathrm{v}$ vaccines over a wide range of ethnic groups. The Japanese population may be of particular interest, because recent $\mathrm{A} / \mathrm{H} 1 \mathrm{~N} 1 \mathrm{v}$ surveillance data show a unique demographic pattern: a low case fatality rate and relatively high percentages of cases and hospitalizations in children. ${ }^{4}$

Limitations in vaccine manufacturing capacity are a major concern during a period of heightened demand, such as during a pandemic. Thus, it is essential to consider alternative routes, such as modern cell cultivation technology, use of adjuvants, and lower antigen 
dose to increase the availability of vaccines for rapid immunization of high-risk populations including children, and to ensure the widest possible spread of the vaccine supply. The cell culture-derived $\mathrm{A} / \mathrm{H} 1 \mathrm{~N} 1 \mathrm{v}$ vaccine in the present study is adjuvanted with MF59, an oil-in-water emulsion that is a component of the seasonal influenza vaccine Fluad ${ }^{\circledR}$ (Novartis Vaccines, Siena, Italy), which has been licensed for use in adults in the European Union (EU) since 1997, and has a confirmed safety profile. ${ }^{24,25}$

We report the results of a randomized, singleblind study (part of a larger clinical program in Japan) to assess the preferred vaccine dose (of antigen and adjuvant) and schedule (one or two doses) of a cell culture-derived A/H1N1v vaccine (Celtura ${ }^{\circledR}$, Novartis Vaccines, Marburg, Germany) on the immunogenicity, safety, and tolerability in a healthy Japanese pediatric population between 6 months and 19 years of age.

\section{MATERIALS AND METHODS}

This multicenter, single-blind, phase $2 / 3$ randomized study was performed in five sites in Japan between October 2009 and December 2009. The study was undertaken in compliance with Good Clinical Practice guidelines and the Declaration of Helsinki. The protocol was approved by all local institutional review boards. Before enrolment, written informed consent was obtained from all parents or legal guardians and the participant, if applicable. This trial was registered at ClinicalTrials.gov: NCT01000207.

\section{Subjects}

A total of 123 healthy Japanese children aged 6 months to 19 years were enrolled. The main exclusion criteria were any history or ongoing (chronic) illness likely to interfere with the results, any history or likelihood of adverse reactions to vaccine components, influenza vaccination (except for seasonal influenza at 1 week or more apart) or influenza infection in the 3 months before enrolment, receipt of any other vaccine from 4 weeks before study to completion of the study, or any known impairment of the immune system. Additional criteria for exclusion consisted of a history of progressive or severe neurologic disorder, a history of clinically suspected development delay, bleeding diathesis, or a surgery planned during the study period that would interfere with the study visit schedule. Negative pregnancy test at day 1 was needed for female participants with childbearing potential who had used birth control measures for at least 2 months before study participation and be committed to using birth control measures for the whole study duration (3 weeks after the second vaccination).

\section{Study Procedures}

The children were randomly assigned in a 1:1 ratio to either group $A$, in which the subjects received the cell-derived $A / H 1 N 1 v$ vaccine containing $3.75 \mu \mathrm{g}$ hemagglutinin antigen (HA) with $50 \%$ of the normal MF59 adjuvant dose, or group B in which the subjects received 7.5 $\mu \mathrm{g} \mathrm{HA}$ with $100 \%$ of the normal MF59 dose, a normal dose being the amount found in one dose of Fluad, the commercially available seasonal influenza vaccine. Two vaccinations were administered 3 weeks apart. Total study duration was 6 weeks with five visits at the clinic. At visit 1 on day 1 (baseline), each subject was given an initial health examination and first blood draw (10 $\mathrm{mL}$ for immunologic and $5 \mathrm{~mL}$ for safety analyses for children aged 3-19 years, $5 \mathrm{~mL}$ each for immunologic and safety analyses, if feasible, for children aged 6-35 months). On the same day, the first dose of vaccine was administered by intramuscular injection in the deltoid muscle 
of the nondominant arm. For children less than 12 months of age, vaccine was administered into the anterolateral aspect of the thigh. At visit 3 on day 22, after a second blood draw, a second dose of the same vaccine was administered in the deltoid muscle of the other arm. A third blood sample was taken at the last visit on day 43 (visit 5). Solicited local and systemic reactions were collected via diary cards for 1 week following the first injection (days 1-7; data collected at visit 2 on day 8 ) and 1 week following the second injection (days 22-28, data collected at visit 4 on day 29). Spontaneous reports of adverse events (AEs) and serious AEs (SAEs) were collected throughout the study period.

\section{Vaccines}

A single vaccine formulation was used, based on $\mathrm{A} / \mathrm{H} 1 \mathrm{~N} 1 \mathrm{v}$ virus cell surface antigen (hemagglutinin) prepared by cell cultivation in Madin-Darby canine kidney cells ${ }^{26}$ from the seed virus A/California/7/2009 (A/H1N1v)-like virus (reassortant virus $X 179 a$ ) supplied by the New York Medical College. This present study is one of the clinical trials that contributed to the regulatory approval of the study vaccine Celtura in Japan. The platform for this drug is the same technology used to produce the seasonal trivalent inactivated subunit vaccine Optaflu ${ }^{\circledR}$ (Novartis Vaccines, Marburg, Germany), which is licensed in the EU in adults older than 18 years. MF59, is an oil-inwater emulsion containing naturally occurring squalene, as used in the commercial seasonal influenza vaccine, Fluad, of which more than 40 million doses have been distributed so far with a confirmed safety profile in adults. ${ }^{24,25}$ A standard dose of MF59, as used in Fluad, contains $9.75 \mathrm{mg}$ squalene, $1.175 \mathrm{mg}$ polysorbate 80 , and $1.175 \mathrm{mg}$ sorbitan trioleate. Vaccines were supplied in monodose, prefilled syringes containing a full dose in $0.5 \mathrm{~mL}$ (7.5 $\mu \mathrm{g}$ HA and full dose of MF59 adjuvant). For the appropriate injection for group A, $0.25 \mathrm{~mL}$ was discarded from the prefilled syringe and the remaining $0.25 \mathrm{~mL}$ was injected into the arm or thigh of the participant, equivalent to $3.75 \mu \mathrm{g}$ HA with half dose of MF59 adjuvant. Children in group B were administered the full $0.5 \mathrm{~mL}$ (7.5 $\mu \mathrm{g}$ HA and full dose of MF59 adjuvant).

\section{Immunogenicity Assessment}

Blood samples taken for immunologic assays at visit 1,3 , and 5 on study day 1,22 , and 43 , respectively, were centrifuged on the same day, and sera were stored at a temperature of $-18^{\circ} \mathrm{C}$ or below and shipped to the Novartis Vaccines Clinical Serology Laboratory in Marburg, Germany, for analysis. Antibody responses were measured by hemagglutinin inhibition (HI) and microneutralization ( $\mathrm{MN}$ ) assays, according to standard methods. ${ }^{27,28} \mathrm{HI}$ antibody responses on days 1, 22, and 43 were expressed as geometric mean titer (GMTs); geometric mean ratio (GMRs) of the postvaccination to prevaccination titer (day 22/day 1 titer and day 43/day 1 titer); seroprotection rates, defined as the percentage of subjects with $\mathrm{HI}$ titers $\geq 40$; and seroconversion rates, defined as percentage of subjects per group achieving at least a fourfold increase in HI titer from a seropositive prevaccination titer $(\geq 10)$ or a rise from $<10$ to $\geq 40$ in those who were originally seronegative. The measures of immunogenicity as determined by MN are illustrated as GMTs, GMRs, percentages with $\mathrm{MN}$ titer $\geq 40$, and rates of fourfold increases.

\section{Safety Assessment}

Subjects were monitored for AEs for 30 minutes immediately after each injection, and local and systemic reactions were recorded 
on a diary card by the parents or legal guardians in the following 7 days until the next study visit. All AEs and medications were collected throughout the entire study period. For children aged 3 to 19 years, the following local reactions were reported via diary card: ecchymosis, erythema, induration, swelling, and pain at injection site. Systemic reactions included headache, arthralgia, chills, fatigue, malaise, myalgia, nausea, sweating, and fever (ie, a temperature $\geq 38^{\circ} \mathrm{C}$ ).

For the infants aged 6 to 35 months, local reactions reported via diary card included ecchymosis, erythema, induration, swelling, and tenderness; systemic reactions reported via diary card included sleepiness, diarrhea, vomiting, irritability, change in eating habits, shivering, unusual crying, and fever. Any other AEs occurring throughout the duration of the study were reported at the subsequent study visit, and SAEs had to be reported immediately to the study sponsor. A physical health assessment was conducted at all visits at the site. Vital signs (blood pressure, pulse, and body temperature) and clinical laboratory tests (hematology, biochemistry, and urinalyses) were assessed at study visit 1,3 , and 5 on day 1,22 , and 43 , respectively.

\section{Statistical Analyses}

The sample size of at least 60 subjects in each group was chosen to exceed the requirements of the European guidelines for yearly influenza vaccine clinical trials of at least 50 subjects per group. ${ }^{29}$ There was no formal statistical hypothesis tested, immunogenicity endpoints being based on HI licensure criteria established by the EU Committee for Medicinal Products for Human Use (CHMP). ${ }^{29,30}$ There are no criteria for immunogenicity in children established by the European Medicines Agency, the closest standards are those used for an adult population. For subjects aged 18 to 60 years, the following criteria apply: (1) the percentage of subjects achieving seroprotection, ie, an HI titer $\geq 40$ is $>70 \%$; (2) percentage of subjects with seroconversion or at least fourfold increase in $\mathrm{HI}$ antibody is $>40 \%$; and (3) the GMR is $>2.5$. Immunogenicity data reflecting the above endpoints as well as the corresponding two-sided 95\% CIs were calculated for each vaccine group. Safety data were evaluated descriptively and expressed as proportions or number of subjects with AEs in each group.

\section{RESULTS}

Of the 123 children enrolled in this study, 122 (99\%) received at least one dose of vaccine and 118 (96\%) received both doses of vaccines. There were 122 and 118 diary cards returned after the first and second vaccinations, respectively. Demographics of the participants are illustrated in Table 1 . The mean age was 8.3 years (range 0-19 years); $51 \%$ of the participants were boys and 49\% were girls. Body composition characteristics and the proportion of subjects

Table 1. Demographics of the study population.

\begin{tabular}{lcc}
\hline & $\begin{array}{c}\text { Group A, } \\
3.75 \mu \mathrm{g}+\mathbf{5 0} \% \\
\text { MF59 }(n=61)\end{array}$ & $\begin{array}{c}\text { Group B, } \\
\mathbf{M F g}+\mathbf{1 0 0 \%} \\
\text { MF59 }(n=62)\end{array}$ \\
\hline Mean \pm SD age, years & $8.0 \pm 4.6$ & $8.6 \pm 5.4$ \\
Boys, \% & 48 & 55 \\
Girls, \% & 52 & 45 \\
Mean \pm SD weight, kg & $29.4 \pm 14.8$ & $30.8 \pm 16.5$ \\
Mean \pm SD height, cm & $124.9 \pm 28.4$ & $126.2 \pm 30.3$ \\
Mean \pm SD BMI, kg/m & $17.5 \pm 2.6$ & $17.6 \pm 2.4$ \\
Prior influenza & 75 & 74 \\
vaccination, \% & & \\
\hline
\end{tabular}

BMI= body mass index; MF59=MF59 adjuvant (Novartis Vaccines, Marburg, Germany). 
who previously received seasonal influenza vaccination (overall 75\%) were similar between the two groups.

\section{Immunogenicity}

HI antibody responses are illustrated in Table 2. At baseline, HI titers against the vaccine strain were low in both study groups, with a GMT of 5.2 for subjects in group A and 6.2 for those in group B. Only four subjects were seroprotected (HI titer $\geq 40$ ) before vaccination. On day 22, 3 weeks after the first vaccination, the cell-derived $\mathrm{A} / \mathrm{H} 1 \mathrm{~N} 1 \mathrm{v}$ vaccine dose containing $7.5 \mu \mathrm{g}$ antigen and

Table 2. Hemagglutinin inhibition (HI) antibody responses of the per protocol set for the two groups at baseline (day 1), 3 weeks after the first vaccination (day 22 ), and 3 weeks after the second vaccination (day 43 ). Numbers in bold represent successful fulfillment of the European Union Committee for Medicinal Products for Human Use (EU CHMP) licensure criteria.

\begin{tabular}{ccc}
\hline & Group A, & Group B, \\
& 3.75 $\mu \mathrm{g}+50 \%$ & $7.5 \mu \mathrm{g}+100 \%$ \\
& MF59 $(n=57)$ & MF59 $(n=59)$ \\
\hline Geometric mean titer $(95 \%$ CI $)$ & \\
Day 1 & $5.2(4.6,6.0)$ & $6.2(5.5,7.1)$ \\
Day 22 & $34(21,54)$ & $84(58,131)$ \\
Day 43 & $355(278,453)$ & $596(471,754)$ \\
\hline
\end{tabular}

Geometric mean ratio (95\% CI)

\begin{tabular}{ccc} 
Day 22 to day 1 & $6.4(4.1,10.0)$ & $13(8.7,21.0)$ \\
Day 43 to day 1 & $68(52,88)$ & $96(75,123)$ \\
\hline Seroprotection rate $($ percentage titer $\geq 40)$ & $(95 \% \mathrm{CI})$ \\
Day 1 & $0(0,6)$ & $7(2,16)$ \\
Day 22 & $56(42,69)$ & $78(65,88)$ \\
Day 43 & $100(94,100)$ & $100(94,100)$
\end{tabular}

Seroconversion rate

(percentage displaying fourfold increase) (95\% CI)

$\begin{array}{lcc}\text { Day } 22 \text { to day } 1 & 56(42,69) & 78(65,88) \\ \text { Day } 43 \text { to day } 1 & 100(94,100) & 100(94,100)\end{array}$

MF59=MF59 adjuvant (Novartis Vaccines, Marburg, Germany).
Figure 1. (A) Percentage of subjects achieving seroprotection (hemagglutinin inhibition [HI] titers 240). (B) Percentage of subjects achieving seroconversion or at least fourfold increase. (C) Geometric means of postvaccination to prevaccination titer ratios (geometric mean ratios [GMRs]). Dotted lines in figures represent the respective European Union Committee for Medicinal Products for Human Use (EU CHMP) licensure criteria for adults under 60 years based on $\mathrm{HI}$ criteria. Day 1 is before vaccination, day 22 is 3 weeks after the first injection, and day 43 is 3 weeks after the second injection. Results are expressed for total age group (6 months-19 years) of the per protocol set. $\mathrm{HA}=$ hemagglutinin antigen. Error bars show 95\% confidence intervals.
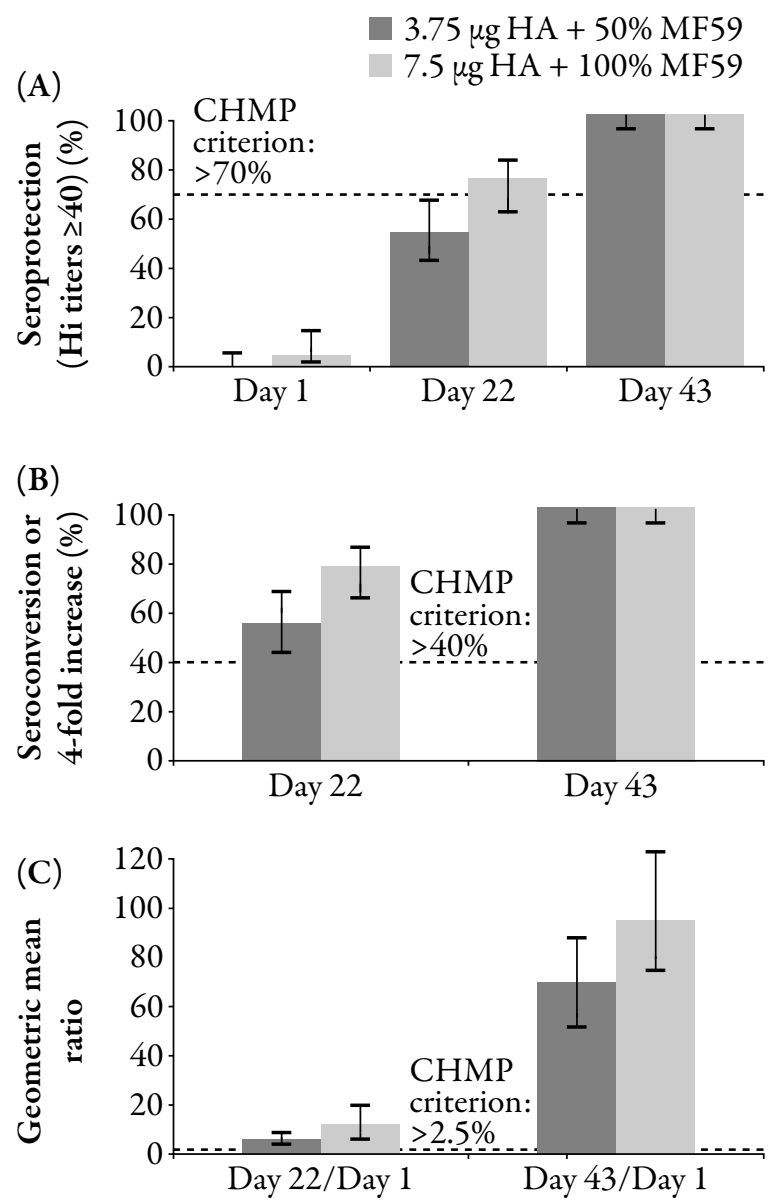

100\% MF59 elicited strong immune responses and met all three EU CHMP criteria, whereas the $3.75 \mu \mathrm{g}$ dose vaccine only fulfilled two of the three criteria. Seroprotection was achieved in $56 \%$ and $78 \%$ of the subjects in groups $\mathrm{A}$ and $\mathrm{B}$, respectively. The seroconversion rates 
of 56\% (group A) and 78\% (group B) and GMRs of 6.4 (group A) and 13 (group B) were all well above the EU CHMP immunogenicity requirements of $40 \%$ and 2.5 , respectively. At day 43, 3 weeks after the second vaccine administration, further increased and robust immunogenic responses were evident in both groups, and all subjects exhibited HI titers $\geq 40$ and seroconversion or a fourfold increase in $\mathrm{HI}$ antibodies, with GMRs of 68 and 96 in groups $A$ and $B$, respectively (Table 2 , Figure 1 ).

A subanalysis of responses in the two groups was performed according to age group (6 to 35 months; 3 to $<9$ years; 9 to 19 years), with the seroprotection rates illustrated in Figure 2. In group B, the youngest (6-35 months) and oldest (9-19 years) age groups who received $7.5 \mu \mathrm{g}$ antigen and 100\% MF59 met all three EU CHMP criteria after the first vaccination (seroprotection rates were $91 \%$ and $79 \%$; seroconversion rates 91\% and 79\%; and GMRs 17 and 13, respectively). Children aged 3 to $<9$ years who received $7.5 \mu \mathrm{g}$ antigen and 100\% MF59 fulfilled two of the three criteria (seroconversion rate of $68 \%$ and GMR 10), the seroprotection rate of $68 \%$ missing the criterion of $70 \%$ (Figure 2). With the lower dose of $3.75 \mu \mathrm{g}$ antigen and $50 \%$ MF59, all age groups fulfilled two of the three criteria after the first vaccination, and further increases in rates of seroprotection and seroconversion were evident 3 weeks after the second vaccination administration, when all subjects in all age groups fulfilled all EU CHMP licensure criteria.

When considered in respect of previous history of influenza vaccination there was no difference observed at day 22, after one vaccination. Seroprotection rates were $46.2 \%$ and $87.5 \%$ for half-dose and full-dose vaccines, respectively, in children with no history of vaccination ( $n=13$ and 16 ), compared with $59.1 \%$ and $74.4 \%$ in those previously vaccinated $(n=44$ and 43 ), $100 \%$ of children being seroprotected at day 43 after the second injection. As previously noted, all subjects had HI titers $\geq 40$ after the second vaccination.

Immunogenicity results as determined by $\mathrm{MN}$ are presented for the total group in Table 3. Overall, robust immunogenic responses in both vaccine groups were evident, confirming the results observed by HI assay. GMTs were low at baseline and increased sixfold to 10-fold after the first vaccination, and 55-fold to 74-fold after the second vaccination in the pooled age groups. The proportions of subjects showing MN titer $\geq 40$ increased from low baseline levels $(0 \%$ and $7 \%)$ to $45 \%$ in subjects in group A and $59 \%$ in those in group B, respectively,

Figure 2. Percentage of subjects achieving seroprotection (hemagglutinin inhibition [HI] titers $\geq 40$ ) in the three age groups (6-35 months; 3 to $<9$ years; 9 to 19 years). Numbers in parentheses are $n$ values for each group. The dotted line shows the $70 \%$ criterion for European Union Committee for Medicinal Products for Human Use (EU CHMP) licensure. Error bars show $95 \%$ confidence intervals. HA=humagglutinin.

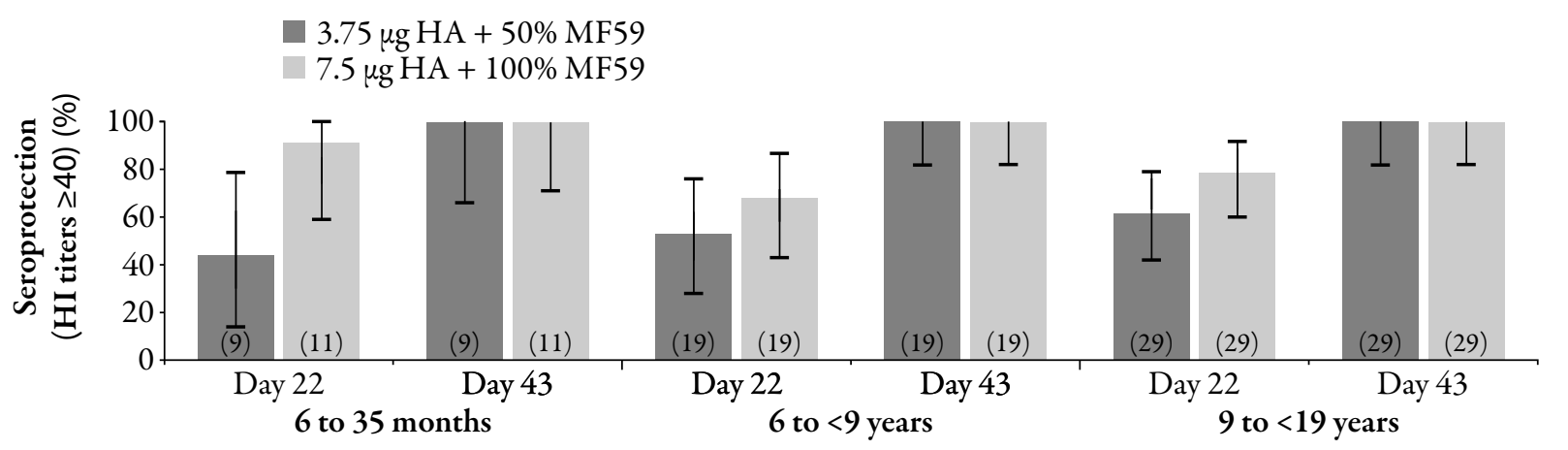


Table 3. Microneutralization (MN) antibody responses of the per protocol set. Results are expressed per vaccine group at baseline (day 1), 3 weeks after the first injection (day 22), and 3 weeks after the second injection (day 43 ).

\begin{tabular}{ccc}
\hline & Group A, & Group B, \\
& $3.75 \mu \mathrm{g}+50 \%$ & $7.5 \mu \mathrm{g}+\mathbf{1 0 0} \%$ \\
& MF59 $(n=57)$ & MF59 $(n=59)$ \\
\hline GMT (95\% CI) & $5.2(4.6,5.8)$ & $6.03(5.4,6.74)$ \\
Day 1 & $31^{*}(20,47)$ & $57 \dagger(38,84)$ \\
Day 22 & $288(226,368)$ & $445 \dagger(351,564)$ \\
Day 43 & $6.0^{*}(4.1,8.9)$ & $9.8 \dagger(6.7,14)$ \\
\hline GMR (95\% CI) & $55(43,71)$ & $74 \dagger(58,94)$ \\
Day 22 to day 1 & $0(0,6)$ & $7(2,16)$ \\
Day 43 to day 1 & $45^{*}(32,59)$ & $59 \dagger(45,71)$ \\
\hline Percentage MN titer $\geq 40, \%(95 \%$ CI $)$ & \\
Day 1 & $98(91,100)$ & $100 \dagger(94,100)$ \\
Day 22 & $60^{*}(46,73)$ & $74 \dagger(61,85)$ \\
Day 43 & $100 \neq(94,100)$ & $100 \dagger(94,100)$ \\
\hline Percentage displaying fourfold increase $(95 \%$ CI $)$ \\
Day 22 to day 1
\end{tabular}

${ }^{*} n=55 ; \dagger n=58 ; \neq n=57$.

GMT=geometric mean titer; GMR=geometric mean of postvaccination to prevaccination titer ratios; MF59=MF59 adjuvant (Novartis Vaccines, Marburg, Germany).

with a further rise to $98 \%$ and $100 \%$ on day 43 . Similarly, a fourfold increase was observed in $60 \%$ (group A) and 74\% (group B) of the subjects on day 22 and 100\% (both groups) on day 43.

\section{Safety}

Local and systemic reactions reported via diary cards during the first 7 days after the first and second vaccinations are presented separately for the relatively small group of children aged 6 to 35 months (Table 4) and the children aged 3 to 19 years (Table 5 ).

For children aged 6 to 35 months, after both the first and second injections $60 \%$ in group $\mathrm{A}$ were reported to have solicited reactions. In group B, 55\% and 36\% were reported to have any solicited reaction after the first and second vaccination doses, respectively. Erythema was the most frequently reported local reaction (27\% to $30 \%$ across groups) and sleepiness was the most frequently reported systemic reaction (up to 30\%), followed by diarrhea and vomiting (both up to $10 \%$ ). Fever (defined as a temperature $\geq 38^{\circ} \mathrm{C}$ ) was present in four subjects in each group, with no reports of fever $\geq 40^{\circ} \mathrm{C}$.

For the older children, aged 3-19 years, $82 \%$ and $84 \%$ of group $\mathrm{A}$, and $92 \%$ and $81 \%$ of group B, were reported to have any solicited reaction after the first and second vaccinations, respectively. In subjects at least 3 years of age, local reactions consisted mainly of pain at the injection site, reported in $64 \%$ and $65 \%$ of group A, and $82 \%$ and $63 \%$ of group B after the first and second vaccinations, respectively, followed by erythema ( $24 \%$ to $41 \%$ across groups) and swelling (27\% to $35 \%$ across groups).

The most frequently reported systemic reactions were fatigue (10\% to $22 \%)$, headache ( $6 \%$ to $22 \%$ ), and malaise (10\% to $21 \%$ ). Fever $\left(\geq 38^{\circ} \mathrm{C}\right)$ was reported in five subjects in group A and in eight subjects in group $\mathrm{B}$, but there were no reports of fever $\geq 40^{\circ} \mathrm{C}$. In both age groups, the frequency and severity of solicited reactions did not increase after the second dose was administered.

There were a few reports of severe reactions on diary cards, consisting of swelling, chills, malaise, headache, fatigue, and nausea, which all resolved within the observation period of 7 days. Use of analgesics was reported in two of the ten 6-35-month-old subjects in group A (20\%) after the second vaccination. In 3-19-year-old children, $10 \%$ and $8 \%$ and $2 \%$ and $6 \%$ of groups $\mathrm{A}$ and $\mathrm{B}$, respectively, reported use of analgesic after the first and second vaccinations. 
Table 4. Solicited local and systemic reactions in 6-35-month-olds after each injection, in each of the vaccine groups.

\begin{tabular}{|c|c|c|c|c|c|}
\hline & \multicolumn{2}{|c|}{ Dose 1} & \multicolumn{2}{|c|}{ Dose 2} \\
\hline & & $\begin{array}{l}3.75 \mu \mathrm{g}, 50 \% \\
\operatorname{MF59}(n=10)\end{array}$ & $\begin{array}{l}7.5 \mu \mathrm{g}, 100 \% \\
\operatorname{MF59}(n=11)\end{array}$ & $\begin{array}{c}3.75 \mu \mathrm{g}, 50 \% \\
\text { MF59 }(n=10)\end{array}$ & $\begin{array}{c}7.5 \mu \mathrm{g}, 100 \% \\
\operatorname{MF} 59(n=11)\end{array}$ \\
\hline \multicolumn{6}{|c|}{ Subjects with local site reactions, \% } \\
\hline \multirow[t]{2}{*}{ Ecchymosis } & Any & 20 & 9 & 10 & 0 \\
\hline & $>100 \mathrm{~mm}$ & 0 & 0 & 0 & 0 \\
\hline \multirow[t]{2}{*}{ Erythema } & Any & 30 & 27 & 30 & 27 \\
\hline & $>100 \mathrm{~mm}$ & 0 & 0 & 0 & 9 \\
\hline \multirow[t]{2}{*}{ Induration } & Any & 20 & 0 & 10 & 9 \\
\hline & $>100 \mathrm{~mm}$ & 0 & 0 & 0 & 0 \\
\hline \multirow[t]{2}{*}{ Swelling } & Any & 10 & 0 & 20 & 18 \\
\hline & $>100 \mathrm{~mm}$ & 0 & 0 & 0 & 9 \\
\hline \multirow[t]{2}{*}{ Tenderness } & Any & 0 & 18 & 20 & 18 \\
\hline & $\begin{array}{l}\text { Cried when injected } \\
\text { limb was moved }\end{array}$ & 0 & 0 & 0 & 0 \\
\hline \multicolumn{6}{|c|}{ Subjects with systemic reactions, $\%$} \\
\hline Sleepiness & Present & 30 & 9 & 10 & 0 \\
\hline Diarrhea & Present & 0 & 9 & 10 & 9 \\
\hline Vomiting & Present & 10 & 9 & 0 & 0 \\
\hline Irritability & Present & 10 & 0 & 0 & 0 \\
\hline Change eating habits & Present & 20 & 0 & 0 & 0 \\
\hline Shivering & Present & 0 & 0 & 0 & 0 \\
\hline Unusual crying & Present & 10 & 0 & 0 & 0 \\
\hline \multirow[t]{2}{*}{ Fever } & $\geq 38.0^{\circ} \mathrm{C}$ & 10 & 18 & 30 & 18 \\
\hline & $\geq 40.0^{\circ} \mathrm{C}$ & 0 & 0 & 0 & 0 \\
\hline \multicolumn{6}{|l|}{ Other } \\
\hline Medication used ${ }^{*}$ & Yes & 0 & 0 & 20 & 0 \\
\hline
\end{tabular}

* Medication used: antipyretics and analgesics.

MF59=MF59 adjuvant (Novartis Vaccines, Marburg, Germany).

Reports of spontaneously occurring AEs were made for $52 \%$ of subjects in group A and $58 \%$ in group B. Of these, $22 \%$ in group A and $29 \%$ in group B were considered at least possibly vaccine related, most of which were classified into general disorders and administrative site conditions (13\% to $18 \%)$, the most frequent being injection site pruritis ( $8 \%$ to $11 \%)$, followed by warmth $(2 \%)$, and pyrexia ( $0 \%$ to $3 \%)$. Additional reactions that were reported include diarrhea, bronchitis, tonsillitis, arthralgia, upper respiratory tract inflammation (all up to 2\%), and ecchymosis, erythema, and rash ( $2 \%$ to $3 \%)$.

Five subjects prematurely withdrew from the study. Two subjects in group B receiving the $7.5 \mu \mathrm{g}$ and $100 \%$ MF59 formulation withdrew due to a mild to moderate $\mathrm{AE}$; one subject reported a rash on study day 1 that resolved after 2 days and whose relationship with the vaccine was not ruled out; the other subject reported bronchitis on study day 17 and persisted at the time of the withdrawal, which was considered 
Table 5. Solicited local and systemic reactions in 3-19-year-olds after each injection, in each of the vaccine groups.

\begin{tabular}{|c|c|c|c|c|c|}
\hline & & \multicolumn{2}{|c|}{ Dose 1} & \multicolumn{2}{|c|}{ Dose 2} \\
\hline & & $\begin{array}{l}3.75 \mu \mathrm{g}, 50 \% \\
\operatorname{MF} 59(n=50)\end{array}$ & $\begin{array}{l}7.5 \mu \mathrm{g}, 100 \% \\
\operatorname{MF} 59(n=51)\end{array}$ & $\begin{array}{l}3.75 \mu \mathrm{g}, 50 \% \\
\operatorname{MF59}(n=49)\end{array}$ & $\begin{array}{c}7.5 \mu \mathrm{g}, 100 \% \\
\text { MF59 }(n=48)\end{array}$ \\
\hline \multicolumn{6}{|c|}{ Subjects with local site reactions, $\%$} \\
\hline \multirow[t]{2}{*}{ Ecchymosis } & Any & 6 & 8 & 4 & 13 \\
\hline & $>100 \mathrm{~mm}$ & 0 & 0 & 0 & 0 \\
\hline \multirow[t]{2}{*}{ Erythema } & Any & 32 & 41 & 24 & 25 \\
\hline & $>100 \mathrm{~mm}$ & 0 & 0 & 0 & 0 \\
\hline \multirow[t]{2}{*}{ Induration } & Any & 18 & 24 & 14 & 17 \\
\hline & $>100 \mathrm{~mm}$ & 0 & 0 & 0 & 0 \\
\hline \multirow[t]{2}{*}{ Swelling } & Any & 28 & 35 & 27 & 33 \\
\hline & $>100 \mathrm{~mm}$ & 0 & 0 & 2 & 0 \\
\hline \multirow[t]{2}{*}{ Pain } & Any & 64 & 82 & 65 & 63 \\
\hline & Severe & 0 & 0 & 0 & 0 \\
\hline \multicolumn{6}{|c|}{ Subjects with systemic reactions, $\%$} \\
\hline \multirow[t]{2}{*}{ Chills } & Any & 4 & 8 & 2 & 6 \\
\hline & Severe & 0 & 4 & 0 & 2 \\
\hline \multirow[t]{2}{*}{ Malaise } & Any & 14 & 16 & 10 & 21 \\
\hline & Severe & 2 & 4 & 2 & 2 \\
\hline \multirow[t]{2}{*}{ Myalgia } & Any & 8 & 18 & 6 & 13 \\
\hline & Severe & 0 & 0 & 0 & 0 \\
\hline \multirow[t]{2}{*}{ Arthralgia } & Any & 2 & 8 & 0 & 2 \\
\hline & Severe & 0 & 0 & 0 & 0 \\
\hline \multirow[t]{2}{*}{ Headache } & Any & 22 & 22 & 6 & 17 \\
\hline & Severe & 2 & 2 & 0 & 2 \\
\hline \multirow[t]{2}{*}{ Sweating } & Any & 2 & 2 & 2 & 0 \\
\hline & Severe & 0 & 0 & 0 & 0 \\
\hline \multirow[t]{2}{*}{ Fatigue } & Any & 16 & 22 & 10 & 13 \\
\hline & Severe & 0 & 2 & 2 & 0 \\
\hline \multirow[t]{2}{*}{ Nausea } & Any & 2 & 8 & 6 & 2 \\
\hline & Severe & 2 & 0 & 0 & 0 \\
\hline \multirow[t]{2}{*}{ Fever } & $\geq 38.0^{\circ} \mathrm{C}$ & 8 & 10 & 2 & 6 \\
\hline & $\geq 40.0^{\circ} \mathrm{C}$ & 0 & 0 & 0 & 0 \\
\hline \multicolumn{6}{|l|}{ Other } \\
\hline Stayed at home & Yes & 4 & 4 & 0 & 4 \\
\hline Medication used ${ }^{*}$ & Yes & 10 & 8 & 2 & 6 \\
\hline
\end{tabular}

${ }^{*}$ Medication used: antipyretics and analgesics.

MF59=MF59 adjuvant (Novartis Vaccines, Marburg, Germany). 
not vaccine related. One subject withdrew from group A with mild pyrexia 3 days after vaccination, which resolved within 3 days. Two other subjects withdrawn from group A were reported with swine influenza and influenza A, respectively. Five SAEs were reported (four cases of influenza and a fracture of the left humerus), none of which were considered related to the study vaccine. The influenza cases involved one subject who was reported to have swine influenza for a duration of 15 days 7 days after receiving their first vaccination with the lowerdose vaccine, and two subjects who received the full dose vaccine who had influenza A for a duration of 4 days from study days 6 and 25, respectively, and one subject given the full dose who had influenza B for a duration of 7 days from study day 20 .

\section{DISCUSSION}

Seasonal influenza and the current 2009 pandemic A/H1N1v influenza are associated with substantial morbidity and hospitalizations in the pediatric population, highlighting the need for effective vaccination in these younger age groups. ${ }^{4,31,32}$ In the present study, we report the results of novel and dose-sparing strategies in influenza vaccine development, on the immunogenicity and safety profile in a healthy Japanese pediatric population aged 6 months to 19 years. Findings of the present study show that one dose of the cell culture-derived $\mathrm{A} / \mathrm{H} 1 \mathrm{~N} 1 \mathrm{v}$ vaccine Celtura, containing $7.5 \mu \mathrm{g}$ of antigen supplemented with the full amount of MF59 adjuvant, elicited robust immunogenic responses in a Japanese pediatric population aged 6 months to 19 years, which met all three EU CHMP licensure criteria. Administration of a half dose of this vaccine $(3.75 \mu \mathrm{g}$ HA together with $50 \%$ of the normal dose of the MF59 adjuvant) fulfilled two of the three EU
CHMP criteria 3 weeks after the first vaccination dose, while a second vaccination resulted in all subjects achieving all three EU CHMP criteria 3 weeks later.

Results expressed for the specific age groups indicate that two doses of vaccine might be needed in children aged 3 to $<9$ years to provide sufficient protection. However, the separate age groups in this study consisted of relatively small numbers of subjects $(n=9-29)$, so these results should be interpreted with caution.

Both doses of vaccine were well tolerated, and the reactogenicity and safety profiles were in line with previous studies using MF59-adjuvanted vaccines in a pediatric population. ${ }^{28,33}$ The majority of AEs were mild to moderate in severity, and none of the five reported SAEs were vaccine related. To elucidate the full safety profile of $\mathrm{A} / \mathrm{H} 1 \mathrm{~N} 1 \mathrm{v}$ vaccines, postmarketing surveillance will be required.

Before the first vaccination, only four subjects exhibited protective HI titers $\geq 40$ against the novel A/H1N1v influenza virus. These low baseline antibody titers are in line with recently published studies from the US and China, ${ }^{12,13}$ but are noticeably lower than the reported percentages from an Australian study, in which $27 \%$ to $33 \%$ of the children aged $\geq 3$ years exhibited seroprotective titers at baseline. Although local A/H1N1v influenza activity was present in Japan during the study, and illustrated by the three subjects who were reported with swine influenza or influenza A, subclinical exposure to the virus was probably ruled out by the stringent exclusion criteria.

Today, both the UK and the US have guidelines that recommend administration of two vaccines for pandemic influenza. ${ }^{34,35}$ However, in line with our findings, recently published trials in adults, the elderly, and children suggest that a single-dose vaccine regimen against the $\mathrm{A} / \mathrm{H} 1 \mathrm{~N} 1 \mathrm{v}$ virus strain may be effective and well 
tolerated. ${ }^{10,11,14,17}$ The pediatric trials that reported satisfactory immune responses after only one dose used a nonadjuvanted split-virion vaccine containing 15 or $30 \mu \mathrm{g}$ of antigen per dose ${ }^{16}$ or an egg-based $\mathrm{A} / \mathrm{H} 1 \mathrm{~N} 1 \mathrm{v}$ vaccine containing 15 or $30 \mu \mathrm{g}$ antigen per dose without adjuvant, or $7.5 \mu \mathrm{g}$ antigen with MF59 adjuvant. ${ }^{17}$ In this latter study, however, only the $7.5 \mu \mathrm{g}$ HA with MF59 adjuvant met the immunogenicity criteria after one dose in children 3 to 8 years of age. ${ }^{17}$ The immunogenic responses observed after one vaccination differ from those of earlier studies in seasonal $\mathrm{H} 1$ strains, and in $\mathrm{H} 5 \mathrm{~N} 1$ vaccination studies where two doses were required to induce sufficient immunogenic protection. ${ }^{19,34}$ Differences may be related to specific antigen characteristics of the new $\mathrm{A} / \mathrm{H} 1 \mathrm{~N} 1 \mathrm{v}$ strain that have yet to be determined. In addition, and particularly in children, the use of appropriate adjuvants may have contributed to the robust immune response observed after one dose of the $7.5 \mu \mathrm{g}$ antigen dose containing the full MF59 dose.

In previous studies, the MF59-adjuvanted influenza vaccine has been shown to provide higher immunogenicity compared with nonadjuvanted vaccines, particularly in those with low prevaccination antibody titers, with a good safety profile in vulnerable populations including the elderly, adults with underlying chronic conditions, and children. ${ }^{21,24,25,28,33}$ In addition, the MF59-adjuvanted vaccines induced broader immunogenicity than conventional vaccines against drifted strains, ${ }^{28,33}$ which may be of particular importance in later phases of a pandemic.

The data from our study do not provide any clear-cut answers for the most effective vaccination strategy in children. However, our findings suggest that one dose of a cellcultivated $\mathrm{A} / \mathrm{H} 1 \mathrm{~N} 1 \mathrm{v}$ vaccine containing $7.5 \mu \mathrm{g}$ HA and $100 \%$ MF59 is effective and well tolerated in a pediatric population, which may have important implications for disease protection and reduced influenza transmission. The use of MF59 adjuvants allows dose sparing, which is an important ethical and moral public health issue to consider in periods of heightened demand. Additional potential advantages of the adjuvant, particularly the enhanced crossreactivity and long-term priming, as previously demonstrated for $\mathrm{H} 5 \mathrm{~N} 1$ vaccines, ${ }^{20,24}$ will only be determined with longer experience of the novel A/H1N1v virus strain.

\section{CONCLUSION}

A single dose of a cell culture-derived A/H1N1v vaccine containing $7.5 \mu \mathrm{g}$ of antigen formulated with the full MF59 adjuvant dose, elicited robust immunogenic responses in a Japanese pediatric population aged 6 months to 19 years, was well tolerated, and met all three EU CHMP licensure criteria. At 3 weeks after the second vaccination dose, subjects who received a half dose of this formulation (3.75 $\mu \mathrm{g}$ of antigen) also fulfilled the three licensure criteria.

\section{ACKNOWLEDGMENTS}

The authors are grateful to all the volunteers who participated in this trial. We acknowledge Dr. PCE de Groot, CHC Europe, for providing support in the manuscript preparation, revision, and editing; collation of author comments and management of the manuscript preparation being performed by K. Veitch (Novartis Vaccines).

Financial Declaration: The study was fully funded by Novartis Vaccines and Diagnostics, the participating investigators receiving fees for expenses incurred in performance of the study, but otherwise have no financial interest in the study data. HS and NN are full-time employees of Novartis Pharma, Japan; RKJ and 
ML are full-time employees of Novartis Vaccines and Diagnostics.

Open Access. This article is distributed under the terms of the Creative Commons Attribution Noncommercial License, which permits any noncommercial use, distribution, and reproduction in any medium, provided the original author(s) and source are credited.

\section{REFERENCES}

1. World Health Organization. DG statement following the meeting of the Emergency Committee. Available at: www.who.int/csr/disease/ swineflu/4th_meeting_ihr/en/index.html. Accessed June 11, 2009.

2. Centers for Disease Control and Prevention. Swine influenza A (H1N1) infection in two children-Southern California, March-April 2009. MMWR Morb Mortal Wkly Rep. 2009;58:400-402.

3. World Health Organization. Pandemic H1N1 2009-Update 86 Geneva. Available at: www.who. int/csr/don/2010_01_29/en/index.html. Accessed January 29, 2010.

4. Kamigaki T, Oshitani H. Epidemiological characteristics and low case fatality rate of pandemic (H1N1) 2009 in Japan. PLoS Curr Influenza. 2009:RRN1139.

5. Centers for Disease Control and Prevention. Serum cross-reactive antibody response to a novel influenza A (H1N1) virus after vaccination with seasonal influenza vaccine. MMWR Morb Mortal Wkly Rep. 2009;58:521-524.

6. Jordan R, Connock M, Albon E, et al. Universal vaccination of children against influenza: are there indirect benefits to the community? A systematic review of the evidence. Vaccine. 2006;24:10471062.

7. Reichert TA, Sugaya N, Fedson DS, Glezen WP, Simonsen L, Tashiro M. The Japanese experience with vaccinating schoolchildren against influenza. N Engl J Med. 2001;344:889-896.

8. Medlock J, Galvani AP. Optimizing influenza vaccine distribution. Science. 2009;325:1705-1708.
9. US Food and Drug Administration, Center for Biological Evaluation and Research. Guidance for industry: clinical data needed to support the licensure of pandemic influenza vaccines. Available at: www.fda.gov/BiologicsBloodVaccines/ GuidanceComplianceRegulatoryInformation/ Guidances/Vaccines/ucm074786.htm. Accessed June 1, 2010.

10. Clark TW, Pareek M, Hoschler K, et al. Trial of 2009 influenza A (H1N1) monovalent MF59-adjuvanted vaccine. N Engl J Med. 2009;361:2424-2435.

11. Greenberg ME, Lai MH, Hartel GF, et al. Response to a monovalent 2009 influenza A (H1N1) vaccine. N Engl J Med. 2009;361:2405-2413.

12. Liang XF, Wang $H Q$, Wang JZ, et al. Safety and immunogenicity of 2009 pandemic influenza A H1N1 vaccines in China: a multicentre, doubleblind, randomised, placebo-controlled trial. Lancet. 2010;375:56-66.

13. Plennevaux E, Sheldon E, Blatter M, ReevesHoche MK, Denis M. Immune response after a single vaccination against 2009 influenza A H1N1 in USA: a preliminary report of two randomised controlled phase 2 trials. Lancet. 2010;375:41-48.

14. Vajo Z, Tamas F, Sinka L, Jankovics I. Safety and immunogenicity of a 2009 pandemic influenza A H1N1 vaccine when administered alone or simultaneously with the seasonal influenza vaccine for the 2009-10 influenza season: a multicentre, randomised controlled trial. Lancet. 2010;375:4955.

15. Zhu FC, Wang H, Fang HH, et al. A novel influenza A (H1N1) vaccine in various age groups. N Engl J Med. 2009;361:2414-2423.

16. Nolan T, McVernon J, Skeljo $M$, et al. Immunogenicity of a monovalent 2009 influenza $\mathrm{A}(\mathrm{H} 1 \mathrm{~N} 1)$ vaccine in infants and children: a randomized trial. JAMA. 2010;303:37-46.

17. Arguedas A, Soley C, Lindert K. Responses to 2009 $\mathrm{H} 1 \mathrm{~N} 1$ vaccine in children 3 to 17 years of age. $\mathrm{N}$ Engl J Med. 2010;362:370-372.

18. Banzhoff A, Gasparini R, Laghi-Pasini F, et al. MF59-adjuvanted H5N1 vaccine induces immunologic memory and heterotypic antibody responses in non-elderly and elderly adults. PLoS One. 2009; $4: \mathrm{e} 4384$.

19. Bernstein DI, Edwards KM, Dekker CL, et al. Effects of adjuvants on the safety and immunogenicity of an avian influenza $\mathrm{H} 5 \mathrm{~N} 1$ vaccine in adults. J Infect Dis. 2008;197:667-675. 
20. Leroux-Roels I, Borkowski A, Vanwolleghem T, et al. Antigen sparing and cross-reactive immunity with an adjuvanted rH5N1 prototype pandemic influenza vaccine: a randomised controlled trial. Lancet. 2007;370:580-589.

21. Stephenson I, Nicholson KG, Hoschler K, et al. Antigenically distinct MF59-adjuvanted vaccine to boost immunity to H5N1. N Engl J Med. 2008;359:1631-1633.

22. Worlth Health Organization. Global alert and response: WHO recommendations on pandemic (H1N1) 2009 vaccines. Available at: www. who.int/csr/disease/swineflu/notes/h1n1 vaccine_20090713/en/index.html. Accessed June 1, 2009.

23. Centers for Disease Control and Prevention. Deaths related to 2009 pandemic influenza A (H1N1) among American Indian/Alaska Natives - 12 states, 2009. MMWR Morb Mortal Wkly Rep. 2009;58:1341-1344.

24. O'Hagan DT. MF59 is a safe and potent vaccine adjuvant that enhances protection against influenza virus infection. Expert Rev Vaccines. 2007;6:699-710.

25. Schultze V, D'Agosto V, Wack A, Novicki D, Zorn J, Hennig R. Safety of MF59 adjuvant. Vaccine. 2008;26:3209-3222.

26. Szymczakiewicz-Multanowska A, Groth N, Bugarini $\mathrm{R}$, et al. Safety and immunogenicity of a novel influenza subunit vaccine produced in mammalian cell culture. J Infect Dis. 2009;200:841-848.

27. Rowe T, Abernathy RA, Hu-Primmer J, et al. Detection of antibody to avian influenza A (H5N1) virus in human serum by using a combination of serologic assays. J Clin Microbiol. 1999;37:937943.
28. Vesikari T, Pellegrini M, Karvonen A, et al. Enhanced immunogenicity of seasonal influenza vaccines in young children using MF59 adjuvant. Pediatr Infect Dis J. 2009;28:563-571.

29. CHMP. Guideline on Influenza Vaccines Pepared from Viruses with the Potential to Cause a Pandemic and Intended for Use Outside of the Core Dossier Context EMEA/CPMP/VWP/263499/2006, 24 January 2007. Available at: www.ema.europa. eu/pdfs/human/vwp/26349906enfin.pdf. Accessed June 1, 2010.

30. CHMP. Guideline on Dossier Structure and Content for Pandemic Influenza Vaccine Marketing Authorisation Application (Revision, EMEA/ CPMP/VEG/4717/2002-Rev1). 18 December 2008. Available at: www.ema.europa.eu/pdfs/human/ vwp/471703enfin.pdf. Accessed June 1, 2010.

31. Neuzil KM, Mellen BG, Wright PF, Mitchel EF, Jr., Griffin MR. The effect of influenza on hospitalizations, outpatient visits, and courses of antibiotics in children. N Engl J Med. 2000;342:225231.

32. Poehling KA, Edwards KM, Weinberg GA, et al. The underrecognized burden of influenza in young children. N Engl J Med. 2006;355:31-40.

33. Vesikari T, Groth N, Karvonen A, Borkowski A, Pellegrini M. MF59-adjuvanted influenza vaccine (FLUAD) in children: safety and immunogenicity following a second year seasonal vaccination. Vaccine. 2009;27:6291-6295.

34. Center for Disease Control and Prevention. Update on influenza A (H1N1) 2009 monovalent vaccines. MMWR Morb Mortal Wkly Rep. 2009;58:11001101.

35. Wise J. Children are likely to need two doses of swine flu vaccine. BMJ. 2009;339:b3969. 